\title{
A Computer Vision System for the Automatic Classification of Five Varieties of Tree Leaf Images
}

\author{
Sajad Sabzi ${ }^{1}\left(\mathbb{D}\right.$, Razieh Pourdarbani $^{1}{ }^{1}$ and Juan Ignacio Arribas ${ }^{2,3, *(\mathbb{C}}$ \\ 1 Department of Biosystems Engineering, College of Agriculture and University of Mohaghegh Ardabili, \\ Ardabil 56199-11367, Iran; sajadsabzi2@gmail.com (S.S.); r_pourdarbani@uma.ac.ir (R.P.) \\ 2 Department of Teoría de la Señal y Comunicaciones e Ingeniería Telemática, University of Valladolid, \\ 47011 Valladolid, Spain \\ 3 Castilla-León Neuroscience Institute (INCYL), University of Salamanca, 37007 Salamanca, Spain \\ * Correspondence: jarribas@tel.uva.es; Tel.: +34-983423000
}

Received: 19 December 2019; Accepted: 24 January 2020; Published: 28 January 2020

\begin{abstract}
A computer vision system for automatic recognition and classification of five varieties of plant leaves under controlled laboratory imaging conditions, comprising: 1-Cydonia oblonga (quince), 2-Eucalyptus camaldulensis dehn (river red gum), 3-Malus pumila (apple), 4-Pistacia atlantica (mt. Atlas mastic tree) and 5-Prunus armeniaca (apricot), is proposed. 516 tree leaves images were taken and 285 features computed from each object including shape features, color features, texture features based on the gray level co-occurrence matrix, texture descriptors based on histogram and moment invariants. Seven discriminant features were selected and input for classification purposes using three classifiers: hybrid artificial neural network-ant bee colony (ANN-ABC), hybrid artificial neural network-biogeography based optimization (ANN-BBO) and Fisher linear discriminant analysis (LDA). Mean correct classification rates (CCR), resulted in $94.04 \%, 89.23 \%$, and 93.99\%, for hybrid ANN-ABC; hybrid ANN-BBO; and LDA classifiers, respectively. Best classifier mean area under curve (AUC), mean sensitivity, and mean specificity, were computed for the five tree varieties under study, resulting in: 1-Cydonia oblonga (quince) 0.991 (ANN-ABC), 95.89\% (ANN-ABC), 95.91\% (ANN-ABC); 2-Eucalyptus camaldulensis dehn (river red gum) 1.00 (LDA), 100\% (LDA), 100\% (LDA); 3-Malus pumila (apple) 0.996 (LDA), 96.63\% (LDA), 94.99\% (LDA); 4-Pistacia atlantica (mt. Atlas mastic tree) 0.979 (LDA), 91.71\% (LDA), 82.57\% (LDA); and 5-Prunus armeniaca (apricot) 0.994 (LDA), 88.67\% (LDA), 94.65\% (LDA), respectively.
\end{abstract}

Keywords: apple; apricot; classification; computer vision; mt. Atlas mastic tree; neural network; precision agriculture; quince; river red gum; site-specific spray

\section{Introduction}

In the past decades, the use of herbicides, pesticides, and other chemical substances has continuously been increased. Unfortunately, overuse of these materials cause surface water pollution, environmental pollution, and animal and human toxicity [1] (Liu and O'Connell, 2002). For this reason, scientists proposed the use of precision agriculture. One of the advantages of precision agriculture is the use of chemical substances only over the area of interest, called site-specific spray. The first step in site-specific spray operations is the proper recognition of the area of interest. Image processing is applied in this field to automatically identify plant species. In the past, there have been various researchers working on the recognition of different plants and trees.

Singh and Bhamrah [2] stated that the recognition of different plants, especially medicine plants is challenging. Eight species of different plants including Amrood, Jaman, Kathal, Nimbu, Palak, Pippal, Sfaida and Toot, were used in this study. From each specimen, ten images were taken. The proposed 
algorithm had five main steps: image histogram equalization, pre-processing, segmentation, feature extraction, and classification. Seven features were extracted from each image to be used in the classifier. Extracted features were: solidity, length of the major axis, length of the minor axis, aspect ratio, area, perimeter, and eccentricity. The classifier used was an artificial neural network. Results showed that the accuracy of this classifier was $98.8 \%$.

Kadir [3] proposed a system to identify some leaves in different plants, based on the combination of Fourier descriptors and some shape features. Extracted features were: translation, scaling, moving the starting point (Fourier descriptors), convexity, irregularity factor, aspect factor, solidity, and roundness factor (shape features). To test the proposed algorithm, a total of 100 images were used. A Bayesian classifier was used. Results showed a classifier accuracy of $88 \%$.

Ehsanirad [4] classified several plant leaves based on texture features such as autocorrelation, entropy, contrast, correlation, and homogeneity. Two methods, gray level co-occurrence matrix (GLCM) and principal component analysis (PCA) were used to feature selection. Following image processing, a total of 65 images from different plants were used to validate and test the system. Results showed a classification accuracy with PCA and GLCM of $98 \%$ and $78 \%$, respectively.

Mursalin et al. [5], performed a study over the classification of five plant species, including Capsicum, Burcucumber, Cogongrass, Marsh herb, and Pigweed. To train and test the proposed system 400 images (80 images from each plant type) were used. Different features were extracted for classification purposes from each image. Extracted features were: solidity, elongatedness, convexity, form factor, area, thickness, perimeter, convex area, convex perimeter, and some color features. Three classifiers were used: support vector machine (SVM), C4.5 and naive Bayes. Results showed the best case of naive Bayes, SVM and C4.5 classifiers with accuracies of 99.3\%, 98.24\%, and 97.86\%, respectively.

In Ahmed et al. [6], authors classified six plants species including Capsicum frutescens L., Amaranthus viridis L., Enhydra fluctuans lour., Chenopodium album L., Imperata cylindrica (L.) P. beauv. and Sicyos angulatus L., based on the SVM classifier. In this study, a total of 224 images were taken from previously listed plants. In the next step, 14 features were extracted from each image in fields of color features, moment invariants, and size-independent shape features. Among previous features, the combination of only 9 features had the best accuracy results. Final nine features used were solidity, elongatedness, mean value component ' $r$ ' in normalized RGB color space, mean value ' $b$ ' in RGB color space, standard deviation ' $r$ ' component, standard deviation of ' $b$ ' component in RGB color space, the $\ln \left(\varphi_{1}\right)$, the $\ln \left(\varphi_{2}\right)$, and the $\ln \left(\varphi_{4}\right)$, of area them all. Results showed that the SVM classifier had an accuracy of $97.3 \%$.

Rumpf et al. [7], used three sequential SVM to classify 10 different plant types including Agropyron repens, Alopecurus Myosuroides, C. Arvense, Galium aparine, Lamium sp., Matricaria inodora, Sinapis arvensis, Stellaria media, Veronica persica, and Hordeum vulgare. From each image, 10 features were extracted: number of pixels of an object, mean distance of the border to the center of gravity, maximum distance of the border to the center of gravity, vertical distance of the border to the main axis of the object, eccentricity, first two-moment invariants, skelmean, and skelsize. Results showed that total accuracy classification for the first SVM classifier was $97.7 \%$, and for the second and third $80 \%$.

Pereira et al. [8], classified three different plant types based on shape features. These plants were: E. crassipes, P. stratiotes, and S. auriculata. In order to train and test the proposed system, a total of 51 images of E. crassipes, 46 images of P. stratiotes and 63 images of S. auriculata were taken. Several features including beam angle statistics, FD, moment invariants, multiscale fractal dimension, and tensor scale descriptor, were extracted. Results showed that a neural network classified these three plant types with an accuracy above $80 \%$.

In addition, Azlah et al. [9], have recently presented a thorough review paper about plant leaf recognition and classification techniques, concluding that the current image processing techniques should be robust under diverse intensity lighting conditions, which could, in turn, be developed by tweaking the detection technique leading to detection of specific plant diseases. 
The aim of this study is to design an intelligent computer vision system to classify five species tree leaves, including 1-Cydonia oblonga (quince), 2-Eucalyptus camaldulensis dehn (river red gum), 3-Malus pumila (apple), 4-Pistacia atlantica (mt. Atlas mastic tree) and 5-Prunus armeniaca (apricot). Figure 1 depicts a flowchart of the computer vision system for plant tree leaves automatic classification, here proposed.
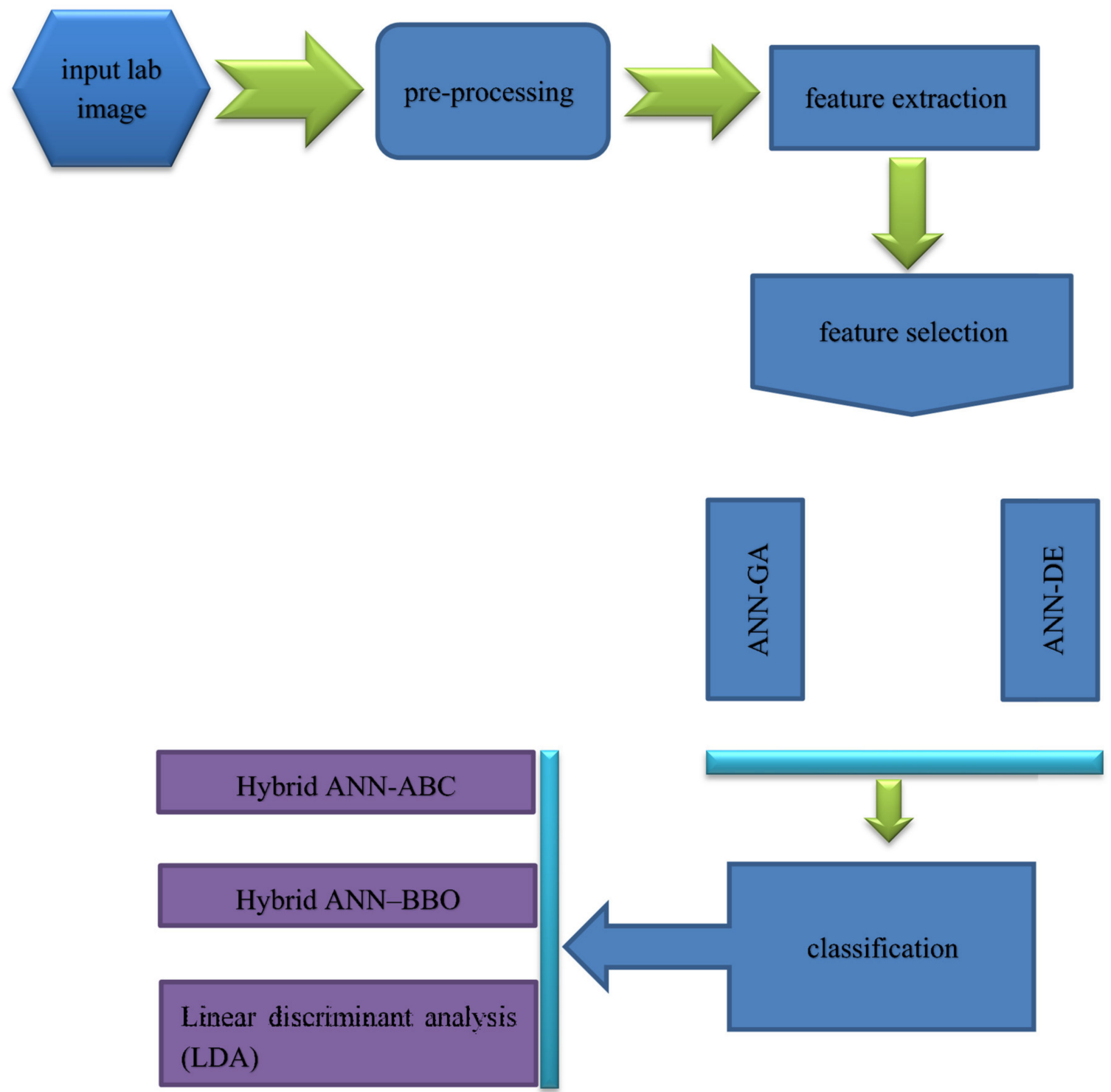

Figure 1. Flowchart of the proposed computer vision system applied in tree leaves automatic classification.

\section{Materials and Methods}

As it can be seen from Figure 1, the proposed computer vision system consists of five steps: the first step includes imaging, the second step includes segmentation and pre-processing, the third step includes feature extraction (including texture descriptors based on the histogram, texture features based on the gray level co-occurrence matrix, shape features, moment invariants, and color features), fourth step includes discriminant feature selection (based on two methods: hybrid artificial neural network-differential evolution (ANN-DE) and hybrid artificial neural network-genetic algorithm (ANN-GA)), and fifth step comprises classification based on three different classifiers, including hybrid artificial neural network-ant bee colony (ANN-ABC), hybrid artificial neural network-biogeography 
based optimization (ANN-BBO) and Fisher's linear discriminant analysis (LDA). Each of the five steps in the here proposed computer vision system, depicted in Figure 1 flowchart, will be further explained with more detail in the next paper sections.

\subsection{Database Used in Computer Vision}

As already mentioned, in this study five different types of leaves were investigated, including 1-Cydonia oblonga (quince), see Appendix A-Eucalyptus camaldulensis dehn (river red gum), see Appendix A-Malus pumila (apple), see Appendix A-Pistacia atlantica (mt. Atlas mastic tree), see Appendix A-Prunus armeniaca (apricot), see Appendix A. Table 1 shows the class number, English common tree name, scientific tree name and the number of samples in each class. Figure 2 shows one sample of each leaf type. All images were taken in Kermanshah, Iran (longitude: $7.03^{\circ} \mathrm{E}$; latitude: $4.22^{\circ} \mathrm{N}$ ). High-quality images were taken with a color GigE industrial camera (ImagingSource, model DFK-23GM021, 1/3 inch Aptina CMOS MT9M021 sensor, 1.2 MP $1280 \times 960$ spatial resolution, Germany), mounting an appropriate Computar CBC Group lens (model H0514-MP2, f = 5 mm F1.4, 1/2 inch type megapixel cameras, Japan). The camera was fixed at $10 \mathrm{~cm}$ above ground level, and all images were taken under white light of 327 lux lighting conditions.

Table 1. Class number, tree common English and scientific names and number of tree leaves images: 516 tree leaves in total.

\begin{tabular}{cccc}
\hline Class \# & English Name & Scientific Name & Number of Samples \\
\hline 1 & quince & Cydonia oblonga & 111 (Figure A1) \\
2 & river red gum & Eucalyptus camaldulensis dehn & 117 (Figure A2) \\
3 & apple & Malus pumila & 101 (Figure A3) \\
4 & mt. Atlas mastic tree & Pistacia atlantica & 89 (Figure A4) \\
5 & apricot & Prunus armeniaca & 98 (Figure A5) \\
\hline
\end{tabular}

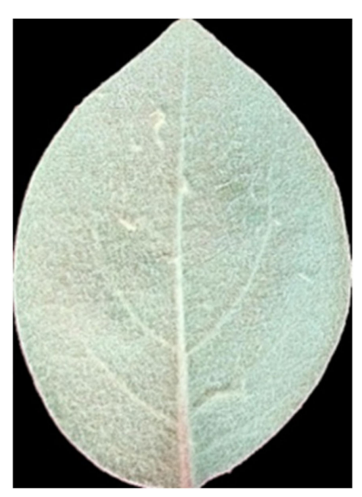

(a)

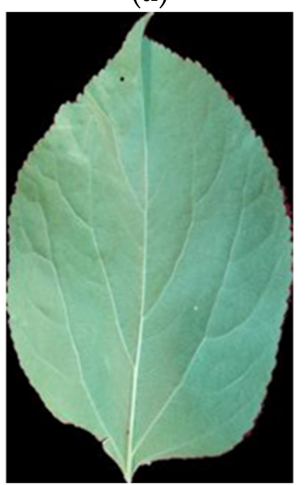

(c)

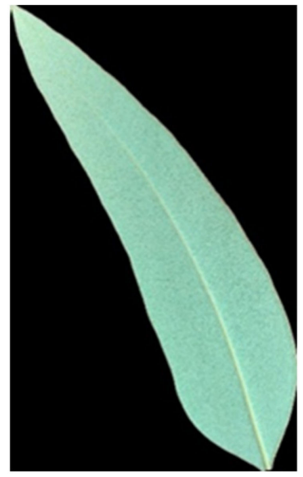

(b)

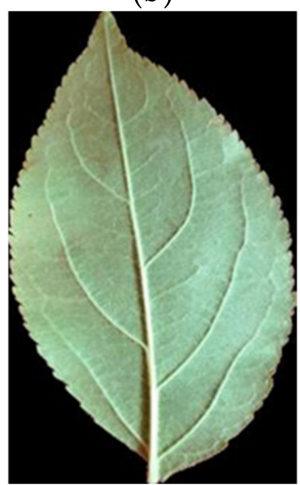

(d)

Figure 2. Cont. 


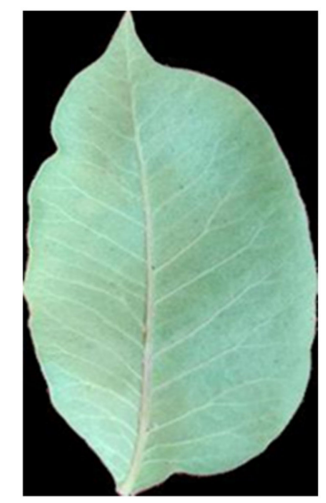

(e)

Figure 2. An image sample of each tree leaf type: (a) 1-Cydonia oblonga (quince), (b) 2-Eucalyptus camaldulensis dehn (river red gum), (c) 3-Malus pumila (apple), (d) 4-Pistacia atlantica (mt. Atlas mastic tree), and (e) 5-Prunus armeniaca (apricot).

\subsection{Image Pre-Processing and Segmentation}

The segmentation stage is an important one in image processing, since in case of wrong segmentation, either the background is considered as an object (in this study, any tree leaf) or object is considered as background. To reach a proper segmentation, six standard color spaces (RGB, HSV, HSI, YIQ, CMY, and YCbCr) were taken into account to find the best color space for segmentation purposes. Results have shown that the best color space for image segmentation was $\mathrm{YCbCr}$ color space and the best channels for image thresholding were luminance $\mathrm{Y}$ and chrominance $\mathrm{Cr}$ channels. Equation (1) was used to set the value of the threshold used to segment objects from their background in tree leaf images:

$$
Y(i, j) \leq 100 \mid C r(i, j) \geq 15 .
$$

The implications of this equation are as follows: each pixel in $\mathrm{YCbCr}$ color space that has $\mathrm{Y}$ components smaller or equal to 100, or Cr components larger or equal to 15 , is considered as background, otherwise, a pixel is considered as foreground (tree leaf object). In order to extract shape features from segmented images, binary images are needed. To extract shape features with high accuracy, some morphological operations are needed, since usually, some "noisy" pixels can exist in segmented images. For this reason, the Matlab imclose function was used, (Gonzalez et al. [10]). Figure 3 summarizes pre-processing operations, shown over three sample tree leaf images. 

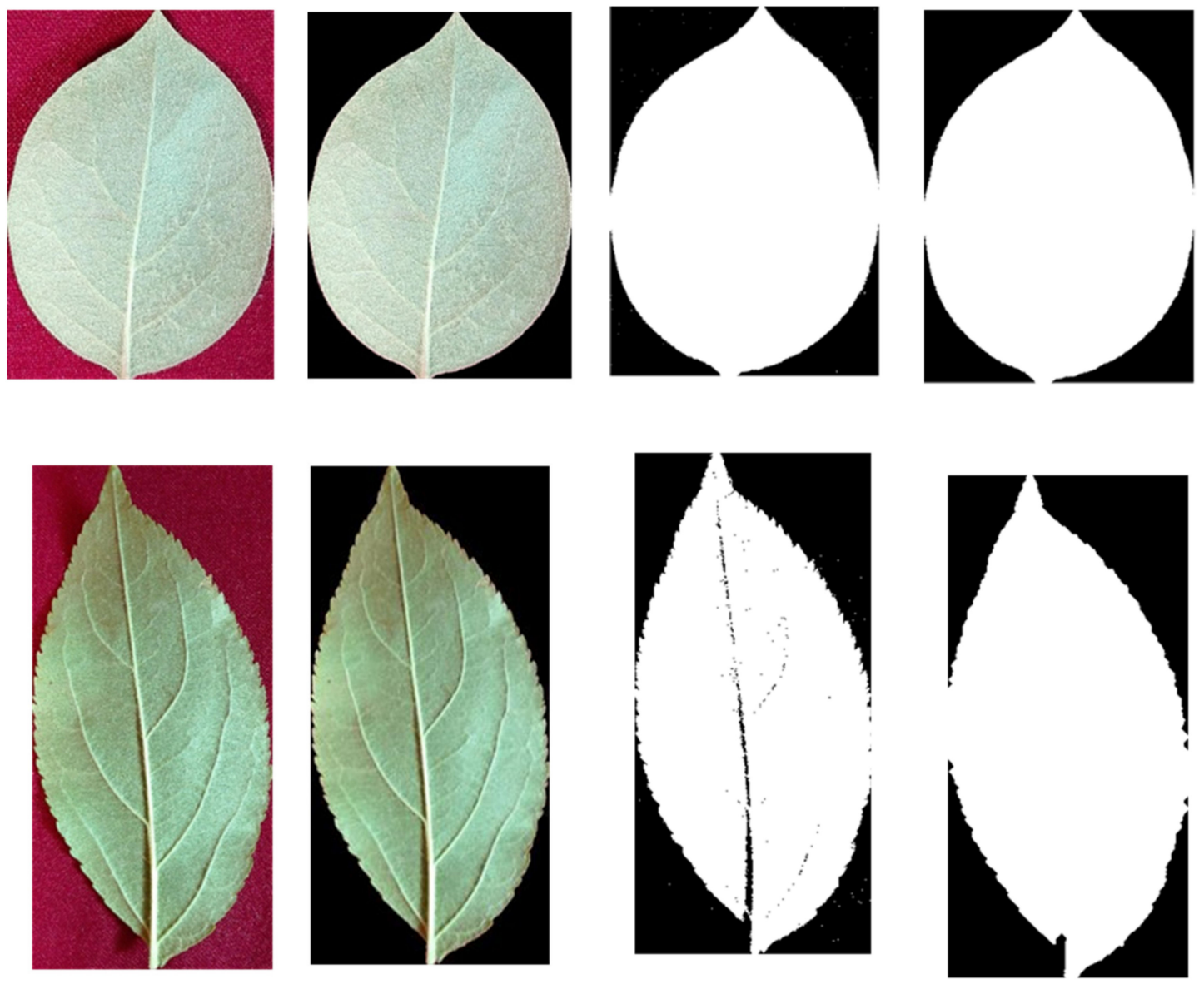

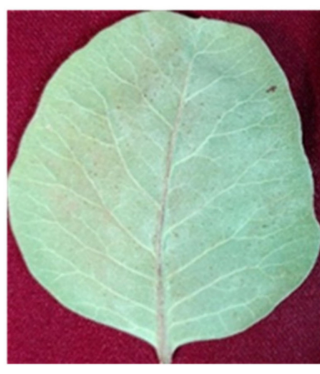

(a)

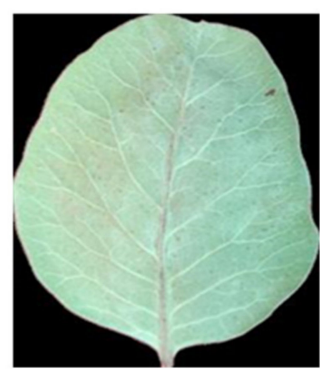

(b)

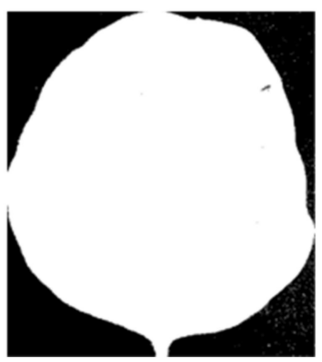

(c)

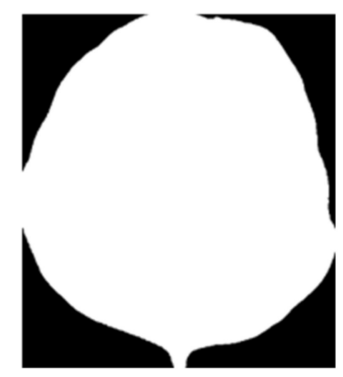

(d)

Figure 3. Three sample tree leaf images used to show image pre-processing and segmentation operations: (a) original tree leaf image, (b) segmented tree leaf image, (c) binary image, and (d) improved binary image.

\subsection{Feature Extraction}

It is well-known that in order to properly classify different tree leaf types, feature extraction is needed. Features of various types and in different fields include texture descriptors based on the histogram, texture features based on the gray level co-occurrence matrix, shape features, moment invariants and color features (others may exist that are not used here). Above mentioned feature types were extracted from each leave. Indeed, the first 285 features of each leaf image were extracted and then effective (high discriminant) features were selected among them by hybrid ANN-DE and hybrid ANN-GA optimization approaches. 


\subsubsection{Texture Descriptors Based on the Histogram}

Texture features that were extracted based on histogram include homogeneity, entropy, smoothness, third moment, average and standard deviation.

\subsubsection{Texture Features Based on the Gray Level Co-Occurrence Matrix (GLCM)}

It is well-known that neighbor angles have a big impact on the estimation of the values of each feature based on the GLCM. Table 2 shows texture features extracted based on the gray level co-occurrence matrix. These features were extracted for four different angles: $0^{\circ}, 45^{\circ}, 90^{\circ}$, and $135^{\circ}$. Thus, in total, $27 \times 4=108$ texture features were extracted from each object.

Table 2. Texture features $(27 \times 4=108)$ extracted based on the gray level co-occurrence matrix $(\mathrm{GLCM})$ : feature number and name.

\begin{tabular}{|c|c|c|c|}
\hline Number & Feature & Number & Feature \\
\hline 1 & contrast & 15 & inverse difference normalized (INN) \\
\hline 2 & sum of squares & 16 & $\begin{array}{l}\text { inverse difference moment } \\
\text { normalized }\end{array}$ \\
\hline 3 & second diagonal moment & 17 & diagonal moment \\
\hline 4 & mean & 18 & sum average \\
\hline 5 & sum entropy & 19 & variance \\
\hline 6 & difference variance & 20 & sum variance \\
\hline 7 & difference entropy & 21 & standard deviation \\
\hline 8 & information measure of correlation 1 & 22 & coefficient of variation \\
\hline 9 & information measure of correlation2 & 23 & maximum probability \\
\hline 10 & $\begin{array}{c}\text { inverse difference (INV) in } \\
\text { homogeneity }\end{array}$ & 24 & Correlation \\
\hline 11 & autocorrelation & 25 & cluster prominence \\
\hline 12 & cluster Shade & 26 & dissimilarity \\
\hline 13 & energy & 27 & entropy \\
\hline 14 & homogeneity & & \\
\hline
\end{tabular}

\subsubsection{Shape Features}

In this study, 29 shape features were extracted. Table 3 lists the shape features that were used here.

Table 3. Shape features (29): feature number and name.

\begin{tabular}{cccc}
\hline Number & Feature & Number & Feature \\
\hline 1 & length & 16 & orientation \\
2 & Euler number & 17 & filled area \\
3 & area & 18 & equiv. diameter \\
4 & logarithm of the ratio of length to width & 19 & width \\
5 & ratio of perimeter to broadness of object & 20 & eccentricity \\
6 & ratio of width to length & 21 & elongation \\
7 & ratio of area to length & 22 & compactness \\
8 & solidity & 23 & extent \\
9 & perimeter & 24 & aspect ratio \\
10 & convex area & 25 & ratio of length to perimeter \\
11 & minor axis length & 26 & perimeter (old way) \\
12 & major axis length & 27 & compactness \\
13 & form factor & 28 & convexity \\
14 & ratio of subtraction and sum of minor axis & 29 & centroid \\
15 & length and major axis length & & \\
\hline
\end{tabular}




\subsubsection{Moment Invariant Features}

Moment invariant features have the advantage that are insensitive to translation, reflection dilation, and rotation. In this study, 10 moment-invariant features were extracted: first-order moment invariant, second-order moment invariant, third-order moment invariant, fourth-order moment invariant, fifth-order moment invariant, sixth-order moment invariant, seventh order moment invariant, difference of first and seventh order moment invariants, difference of second and sixth-order moment invariants and difference of third and fifth-order moment invariants.

\subsubsection{Color Features}

Different color features have different values in different color spaces. For this reason, some color features were extracted in RGB, YCbCr, YIQ, CMY, HSV and HSI color spaces. Extracted color features are divided into two groups:

1. 'Statistical' color features

2. Vegetation index color features.

\section{'Statistical' Color Features}

These features comprised mean and standard deviations of the first color component, the second component, third component and mean of first, second and third components for all RGB, YCbCr, YIQ, CMY, HSV, and HSI color spaces. Thus, the total number of 'statistical' color features was $2 \times 4 \times 6=48$.

\section{Vegetation Index Color Features}

Table 4 lists 14 vegetation index features for RGB color space, including mathematical definitions. These features were also computed for the other five color spaces (YCbCr, YIQ, CMY, HSV, and HSI) totaling $14 \times 6=84$ vegetation index features.

Table 4. Vegetation index features $(14 \times 6=84)$ : feature name and mathematical formulae definitions.

\begin{tabular}{|c|c|}
\hline Feature Name & Mathematical Equation Definition \\
\hline normalized first component of RGB color space & $R_{n}=R /(R+G+B)$ \\
\hline normalized second component of RGB color space & $G_{n}=G /(R+G+B)$ \\
\hline normalized third component of RGB color space & $B_{n}=B /(R+G+B)$ \\
\hline gray channel & gray $=0.2898 \times R_{n}+0.5870 \times G n+0.1140 \times B_{n}$ \\
\hline additional green (Woebbecke et al. [11]) & $E X G=2 \times G_{n}-R_{n}-B_{n}$ \\
\hline additional red (Meyer et al. [12]) & $E X R=1.4 \times R_{n}-G_{n}$ \\
\hline color index for vegetation cover (Kataoka et al. [13]) & $C I V E=0.441 \times R_{n}-0.811 \times G_{n}+0.385 \times B_{n}+18.78$ \\
\hline $\begin{array}{l}\text { subtraction between additional green and additional } \\
\text { red (Meyer and Neto [14]) }\end{array}$ & $E X G R=E X G-E X R$ \\
\hline normalized difference index (Woebbecke et al. [15]) & $N D I=\left(G_{n}-B_{n}\right) /\left(G_{n}+B_{n}\right)$ \\
\hline green minus blue index (Woebbecke et al. [11]) & $G B=\left(G_{n}-B_{n}\right)$ \\
\hline red-blue contrast (Golzarian and Frick [16]) & $R B I=\left(G_{n}-B_{n}\right) /\left(G_{n}+B_{n}\right)$ \\
\hline green-red index (Golzarian and Frick [16]) & $E R I=\left(R_{n}-G_{n}\right) \times\left(R_{n}-B_{n}\right)$ \\
\hline additional green index (Golzarian and Frick [16]) & $E G I=\left(G_{n}-R_{n}\right) \times\left(G_{n}-B_{n}\right)$ \\
\hline additional blue index (Golzarian and Frick [16]) & $E B I=\left(B_{n}-G_{n}\right) \times\left(B_{n}-R_{n}\right)$ \\
\hline
\end{tabular}

\subsection{Discriminant Feature Selection}

As mentioned before, 285 features were extracted from each leaf image object. The use of all features as input to classifiers is not wise, given the problem of overfitting and poor generalization to the test set. The selection of discriminant groups of features is a well-known good practice. To do so, two methods based on artificial intelligence were used to select effective features (results to be shown later on): 
1. ANN-DE

2. ANN-GA.

\subsection{Optimal Neural Network Classifier Parameters}

Classification is the final step in designing a computer or machine vision system. A highperformance classifier guarantees the accuracy of the computer version system. In this study, three classifiers including hybrid ANN-ABC, hybrid ANN-BBO and Fisher's linear discriminant analysis (LDA), were used in classifying. In order to have statistically valid results, 100 uniform random simulations were repeated. All data were divided into two disjoint groups, training, and validation set data (60\% input samples) and test set data (40\% of input samples) in each of the 100 averaged simulations, following a uniform random distribution with probability 0.6 and 0.4 to belong to train/validation and test sets, respectively. It is worth mention that a multilayer perceptron (MLP) ANN was used. An MLP neural network has five adjustable parameters: number of neural network layers, number of neurons in each layer, nonlinear transfer function, back-propagation network training function, and back-propagation weight/bias learning function. $\mathrm{ABC}$ and $\mathrm{BBO}$ algorithms were used to select optimal MLP parameter values. Table 5 shows the optimum values of the MLP parameters which were determined with $\mathrm{ABC}$ and $\mathrm{BBO}$ algorithms.

Table 5. Optimal neural network parameters under MatLab, as determined by ant bee colony (ABC) and BBO algorithms. satlin: saturating linear transfer function; tribas: triangular basis transfer function; tansig: hyperbolic tangent sigmoid transfer function; purelin: linear transfer function; logsig: log-sigmoid transfer function; trainlm: Levenberg-Marquardt backpropagation learning rule; learnwh: Widrow-Hoff weight/bias learning function; learncon: conscience bias learning function.

\begin{tabular}{|c|c|c|c|c|c|}
\hline Algorithm & $\begin{array}{l}\text { Number of } \\
\text { Layers }\end{array}$ & $\begin{array}{l}\text { Number of } \\
\text { Neurons }\end{array}$ & $\begin{array}{l}\text { Nonlinear Transfer } \\
\text { Functions }\end{array}$ & $\begin{array}{c}\text { Backpropagation } \\
\text { Network Training } \\
\text { Function }\end{array}$ & $\begin{array}{l}\text { Backpropagation } \\
\text { Weight/Bias } \\
\text { Learning Function }\end{array}$ \\
\hline $\mathrm{ABC}$ & 3 & $\begin{array}{l}\text { First layer: } 25, \\
\text { second layer: } 25 \text {, } \\
\text { third layer: } 25\end{array}$ & $\begin{array}{l}\text { First layer: satlin, } \\
\text { second layer: tribas, } \\
\text { third layer: tansig }\end{array}$ & trainlm & learnwh \\
\hline $\mathrm{BBO}$ & 3 & $\begin{array}{l}\text { First layer: } 17, \\
\text { second layer: } 16 \text {, } \\
\text { third layer: } 15\end{array}$ & $\begin{array}{l}\text { First layer: satlin, } \\
\text { second layer: purelin, } \\
\text { third layer: logsig }\end{array}$ & trainlm & learncon \\
\hline
\end{tabular}

\section{Results and Discussion}

To evaluate the three different classifiers' performance (hybrid ANN-ABC, hybrid ANN-BBO and LDA), various criteria are computed, including confusion matrix, sensitivity, specificity and accuracy, receiver operating characteristic (ROC) and area under the ROC curve (AUC). Before the evaluation of the classifiers' performance, selected features based on ANN-DE and ANN-GA approaches are shown next.

\subsection{Feature Selection Based on ANN-DE and ANN-GA Approaches}

As mentioned in previous sections, two ANN-DE and ANN-GA approaches were used to select effective (discriminant) features. Table 6 shows the seven selected features by ANN-DE and ANN-GA. As one can see, ANN-DE method selected all features among shape and texture features based on the gray level co-occurrence matrix, while the ANN-GA method selects discriminant features among shape, texture based on the gray level co-occurrence matrix and color features. Figures 4 and 5 show boxplot graphs for the value of each extracted feature by ANN-DE and ANN-GA methods, respectively. Figure 4 (ANN-DE) shows that quince, red gum, mastic tree, and apricot classes can be separated based on only three features, inverse difference moment normalized related to 0 degrees (IDMN_0), homogeneity related to 0 degree (homogeneity_0) and mean related to 45 degrees (mean_45), since those features have the lowest overlapping boxplots among classes. The same can be said, for obvious 
reasons, about Figure 5c (ANN-GA) regarding homogeneity related to 0 degrees (homogeneity_0) feature. Figure 6 shows the validation step, where we plot mean square error (MSE) as a function of iteration number while learning for hybrid ANN-ABC, hybrid ANN-BBO and LDA classifiers, based on the selected features obtained with ANN-DE and ANN-GA methods listed above. Figure 6a-c show that MSE for selected features based on ANN-DE are lower than those MSE for selected features based on ANN-GA. Having said that, selected features by ANN-DE were finally used as input in getting the classification results.

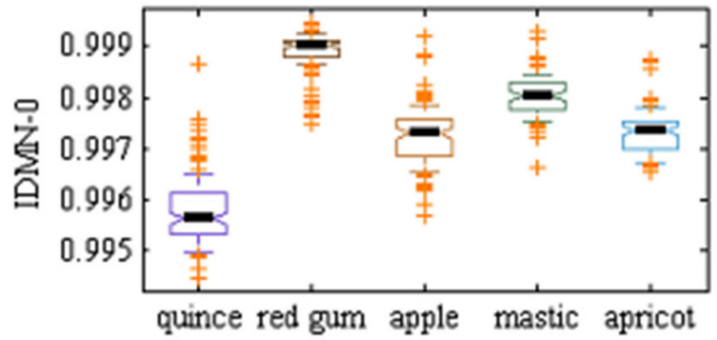

(a)

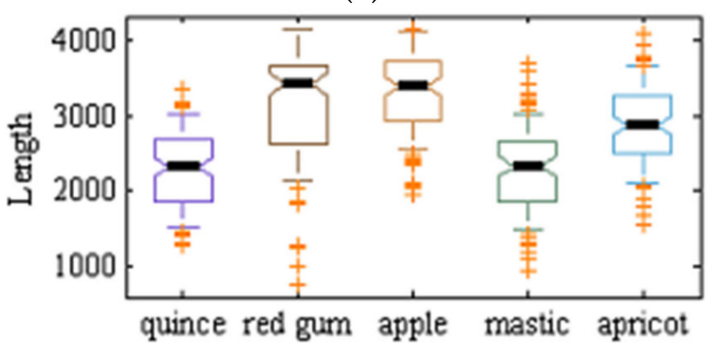

(c)

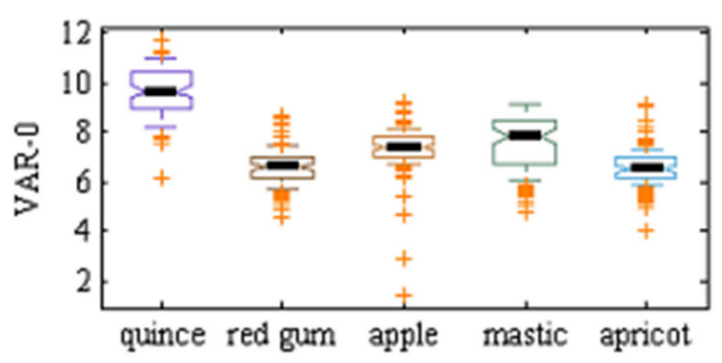

(e)

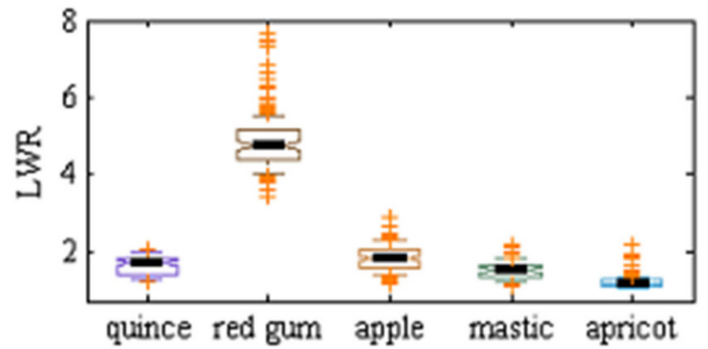

(b)

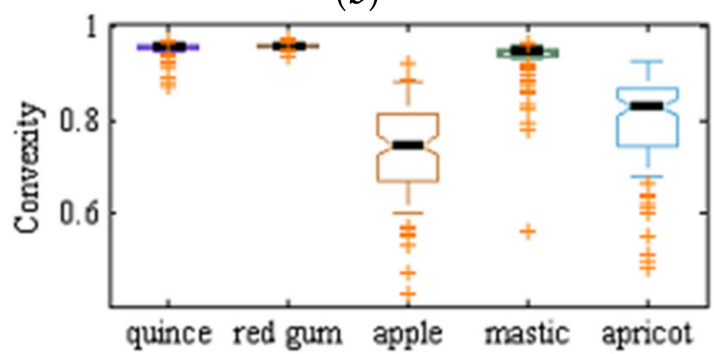

(d)

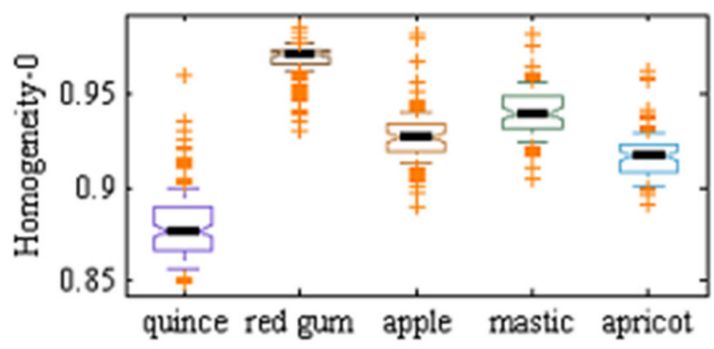

(f)

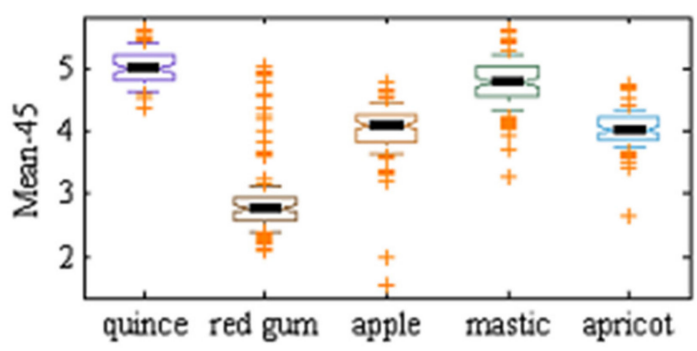

(g)

Figure 4. Boxplot graphs for the value of each of the seven selected features by artificial neural network-differential evolution (ANN-DE) algorithm over the five tree varieties under study: (a) IDMN_0, (b) LWR, (c) length, (d) convexity, (e) VAR_0, (f) homogeneity_0, and (g) mean_45 (cf. Table 6). 


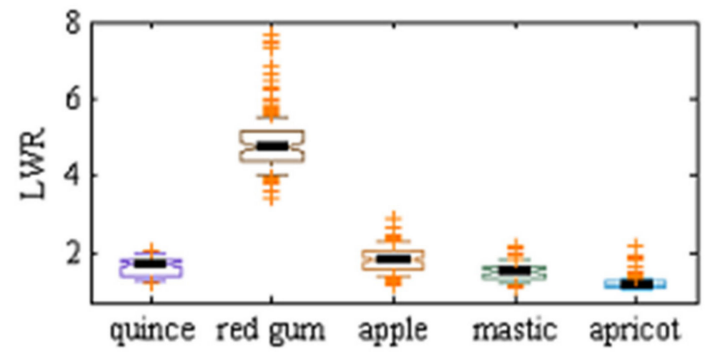

(a)

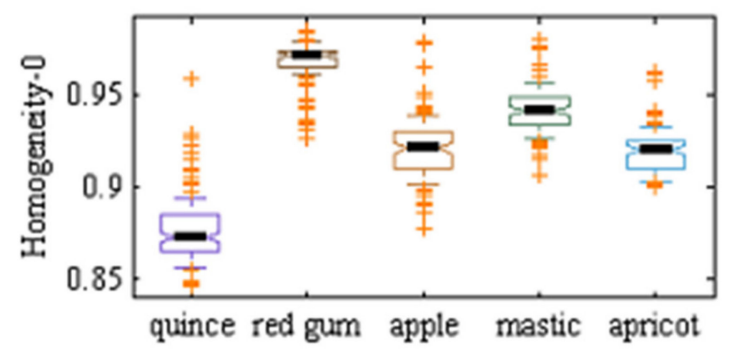

(c)

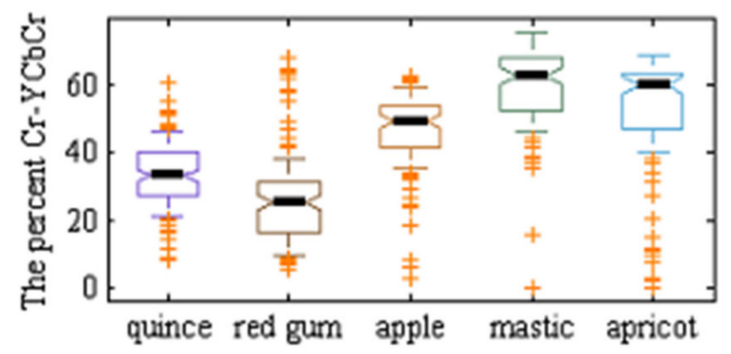

(e)

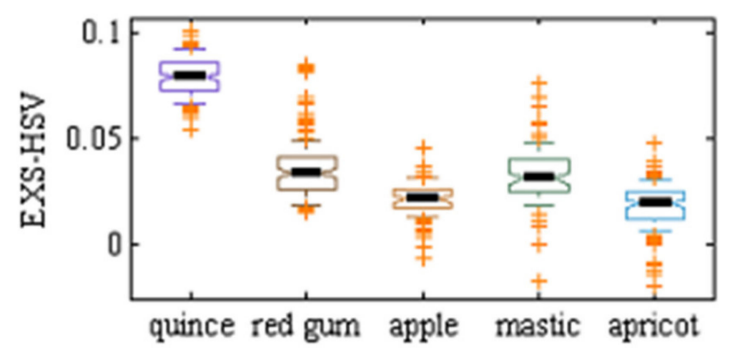

(b)

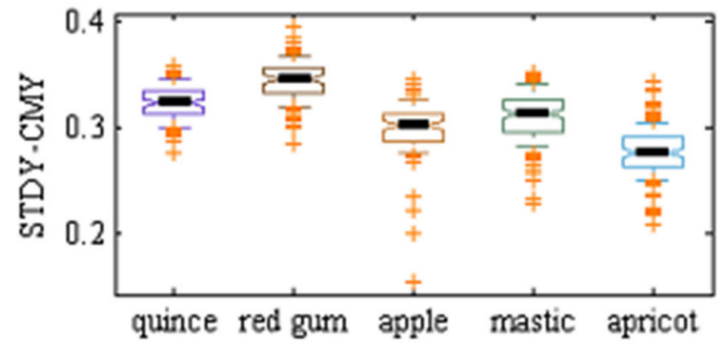

(d)

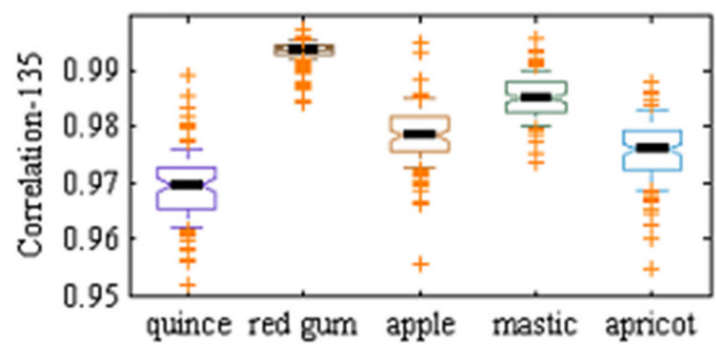

(f)

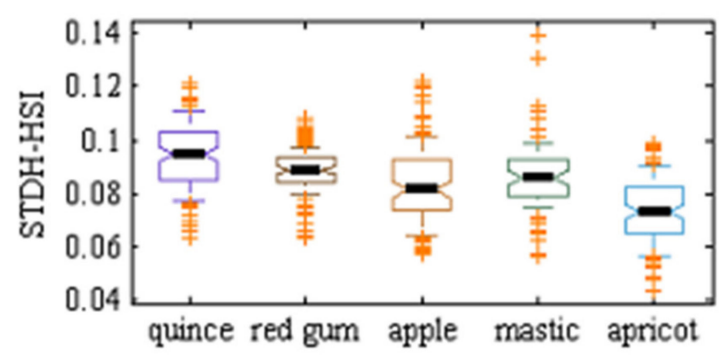

(g)

Figure 5. Boxplot graphs for the value of each of the seven selected features by the artificial neural network-genetic algorithm (ANN-GA) algorithm over the five tree varieties under study: (a) LWR, (b) EXS_HSV, (c) homogeneity_0, (d) STDY_CMY, (e) percentage Cr-YCbCr, (f), correlation_135, and (g) STDH_HSI (cf. Table 6). 
Table 6. Discriminant feature selection based on the ANN-DE and ANN-GA methodologies: seven final discriminant features selected in each method.

\begin{tabular}{|c|c|}
\hline Method & Selected Features \\
\hline \multirow{7}{*}{ ANN-DE } & inverse difference moment normalized related to 0 degree (IDMN_0) \\
\hline & ratio of length to width (LWR) \\
\hline & length \\
\hline & convexity \\
\hline & variance related to 0 degree (VAR_0) \\
\hline & 6. homogeneity of 0 degree (Homogeneity_0) \\
\hline & mean of 45 degrees (Mean_45) \\
\hline \multirow{7}{*}{ ANN-GA } & 1. ratio of length to width (LWR) \\
\hline & 2. additional second component in HSV color space index (EXS_HSV) \\
\hline & homogeneity of 0 degree (Homogeneity_0) \\
\hline & 4. standard deviation of the third component of CMY color space (STDY_CMY) \\
\hline & $\begin{array}{l}\text { 5. percentage of pixels between levels } 100 \text { and } 120 \text { of third component in } \mathrm{YCbCr} \text { color } \\
\text { space (Percentage } \mathrm{Cr}-\mathrm{YCbCr} \text { ) }\end{array}$ \\
\hline & 6. correlation of 135 degrees (Correlation_135) \\
\hline & 7. standard deviation of first component of HSI color space (STDH_HSI) \\
\hline
\end{tabular}

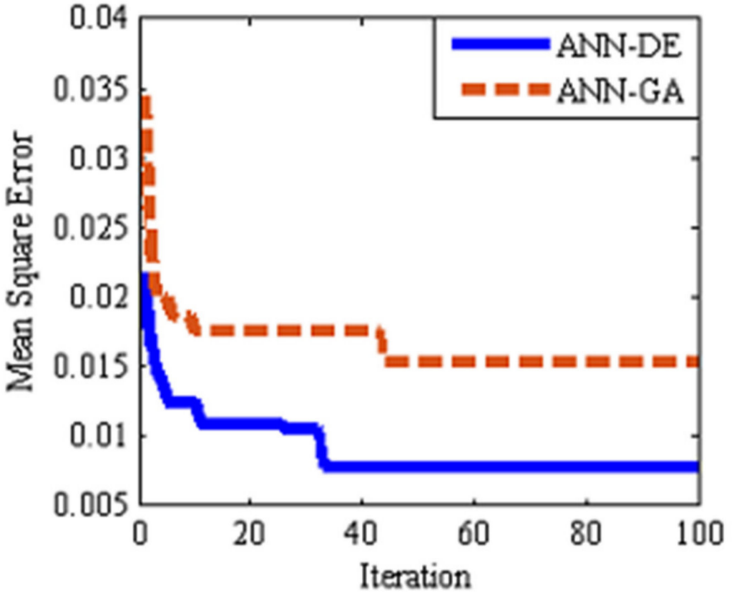

(a)

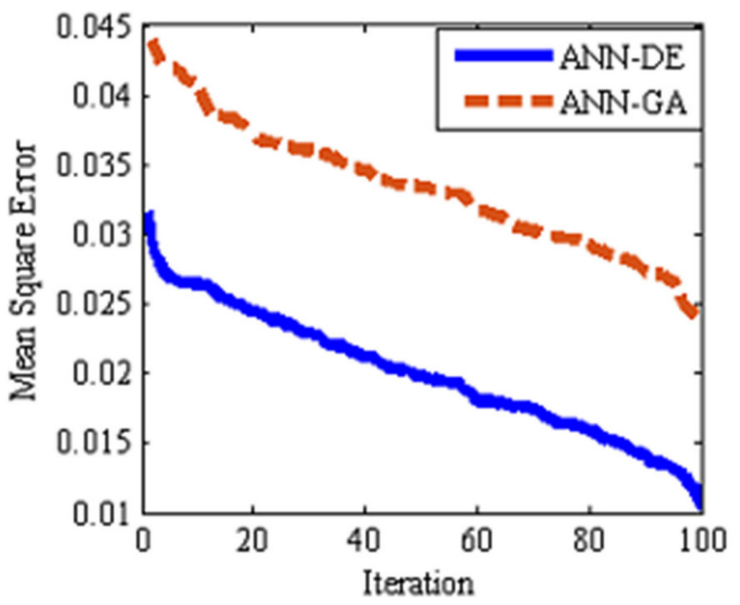

(c)

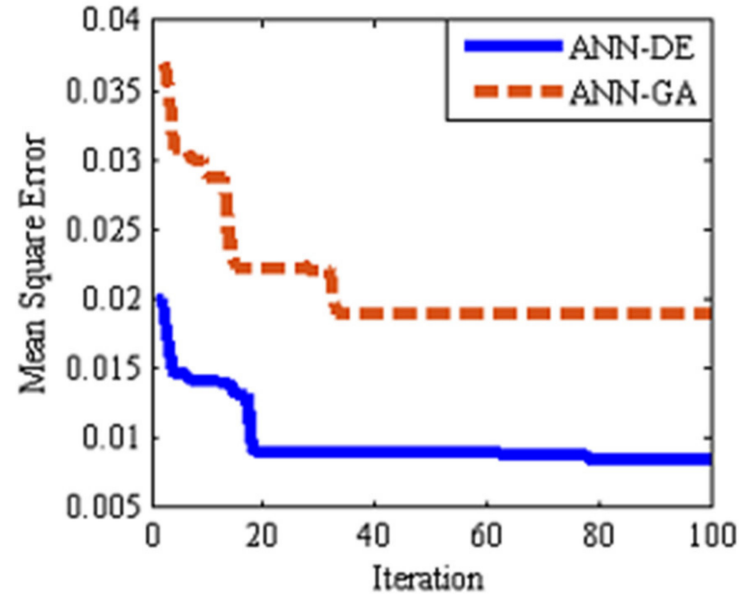

(b)

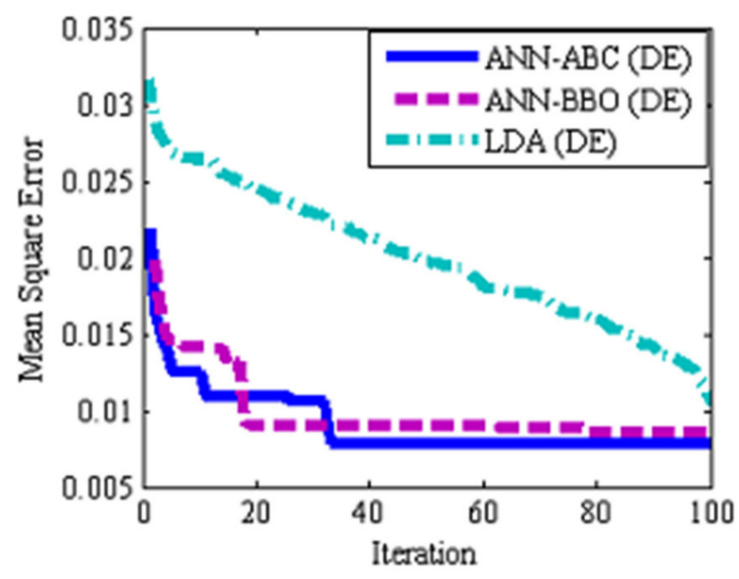

(d)

Figure 6. Mean square learning (train) error as a function of iteration number using ANN-DE and ANN-GA feature selection algorithms: (a) ANN-ABC classifier, (b) artificial neural network-biogeography based optimization (ANN-BBO) classifier, (c) LDA classifier, and (d) best performance among all three classifiers (ANN-DE). 


\subsection{Classification Based on Hybrid ANN-ABC, Hybrid ANN-BBO and LDA}

In this study, three classifiers were used: hybrid ANN-ABC, hybrid ANN-BBO and LDA. To achieve statistical valid results, we averaged 100 simulations with uniform random train and test sets; the probability of an input sample to belong to the train set was 0.6 and 0.4 to belong to the test set.

\subsubsection{Classification Based on the Hybrid ANN-ABC}

Table 7 shows confusion matrix classification results, using ANN-ABC classifier for 100 iterations, over the test set. The lowest classifier error value is that of red gum class with only 17 misclassified samples among 4703 total test samples, resulting in accuracy above $99.6 \%$ for this class. The highest classifier error value is given in the mastic tree class, where 453 out of 3542 samples were misclassified, resulting in a misclassification rate of $12.79 \%$. Figure 2 shows that the shape of 2 -Eucalyptus camaldulensis dehn samples (river red gum) are very different from other class samples, so samples related to this class are classified with high accuracy. On the other hand, as a possible explanation to previous facts, Figure 2 shows how river red gum tree leaves are quite different in shape to other four tree leaves species, which at the same time have quite similar shapes among them, thus resulting in most misclassification errors among these four classes. In summary, the ANN-ABC classifier classified the 20,700 test samples with an accuracy above $94 \%$, which has to be judged as very good for our tree leaf image database.

Table 7. Confusion matrix classification results for the hybrid ANN-ABC classifier: 100 random train and test set samples, results computed over the test set. T: true class, E: estimated class.

\begin{tabular}{ccccccccc}
\hline T $\backslash \mathbf{E}$ & Quince & Red Gum & Apple & Mastic & Apricot & All Data & $\begin{array}{c}\text { Partial Incorrect } \\
\text { Classification } \\
\text { Rate (\%) }\end{array}$ & $\begin{array}{c}\text { Correct } \\
\text { Classification } \\
\text { Rate (\%) }\end{array}$ \\
\hline quince & 4273 & 0 & 9 & 158 & 16 & 4456 & 4.11 & \\
red gum & 1 & 4686 & 2 & 14 & 0 & 4703 & 0.362 & \\
apple & 33 & 23 & 3792 & 117 & 86 & 4051 & 6.39 & 94.04 \\
mastic & 118 & 8 & 114 & 3089 & 213 & 3542 & 12.79 & \\
apricot & 30 & 10 & 93 & 190 & 3625 & 3948 & 8.18 & \\
\hline
\end{tabular}

\subsubsection{Classification Based on the Hybrid ANN-BBO}

Table 8 shows confusion matrix classification results using hybrid ANN-BBO classifier for 100 iterations averaged, over the test set. As can be seen, 2230 samples from 20,700 samples were misclassified by this classifier $(10.77 \%)$. Again, the lowest ANN-BBO classifier accuracy is for mastic tree $(82.20 \%)$ and the highest classifier accuracy is again for river red gum tree $(94.60 \%)$, similar to the hybrid ANN-ABC classifier, and considered as promising results, despite on average the ANN-BBO classifier performance is behind its partners.

Table 8. Confusion matrix classification results for the hybrid ANN-BBO classifier: 100 random train and test set samples, results computed over the test set. T: true class, E: estimated class.

\begin{tabular}{|c|c|c|c|c|c|c|c|c|}
\hline $\mathbf{T} \backslash \mathbf{E}$ & Quince & Red Gum & Apple & Mastic & Apricot & All Data & $\begin{array}{l}\text { Partial Incorrect } \\
\text { Classification } \\
\text { Rate }(\%)\end{array}$ & $\begin{array}{c}\text { Correct } \\
\text { Classification } \\
\text { Rate }(\%)\end{array}$ \\
\hline quince & 4132 & 55 & 66 & 147 & 75 & 4475 & 7.66 & \\
\hline red gum & 106 & 4326 & 52 & 62 & 27 & 4573 & 5.40 & \\
\hline apple & 140 & 58 & 3573 & 145 & 162 & 4078 & 12.38 & 89.23 \\
\hline mastic & 196 & 55 & 131 & 2913 & 249 & 3544 & 17.80 & \\
\hline apricot & 87 & 17 & 168 & 232 & 3526 & 4030 & 12.51 & \\
\hline
\end{tabular}

\subsubsection{Classification Based on LDA}

Table 9 shows classification results presented as a confusion matrix for the LDA classifier for 100 uniform random train and test set iterations averaged, over the test set. The interesting thing here is 
that the LDA classifier classified all samples in the river red gum class correctly. So it can be concluded that for easy class data, the LDA classifier has higher accuracy. On the other hand, the LDA classifier has less accuracy than the hybrid ANN-ABC classifier in quince and apricot classes. Finally, one can see how LDA classifier classified all test data with an overall accuracy of $93.99 \%$, that is high accuracy very close to the $94.04 \%$ overall accuracy for the ANN-ABC classifier, and both clearly over the $89.23 \%$ overall accuracy for the ANN-BBO classifier, which performed the poorest of the three.

Table 9. Confusion matrix classification results for LDA classifier: 100 random train and test set samples, results computed over the test set. T: true class, E: estimated class.

\begin{tabular}{ccccccccc}
\hline $\mathbf{T} \backslash \mathbf{e}$ & Quince & Red gum & Apple & Mastic & Apricot & All Data & $\begin{array}{c}\text { Partial Incorrect } \\
\text { Classification } \\
\text { Rate (\%) }\end{array}$ & $\begin{array}{c}\text { Correct } \\
\text { Classification } \\
\text { Rate (\%) }\end{array}$ \\
\hline quince & $\mathbf{4 1 3 0}$ & 0 & 0 & 366 & 0 & 4496 & 8.14 & \\
red gum & 0 & $\mathbf{4 6 5 8}$ & 0 & 0 & 0 & 4658 & 0 & \\
apple & 42 & 0 & $\mathbf{3 9 2 7}$ & 85 & 10 & 4064 & 3.37 & 93.99 \\
mastic & 22 & 0 & 85 & $\mathbf{3 2 5 1}$ & 187 & 3545 & 8.29 & \\
apricot & 89 & 0 & 122 & 235 & $\mathbf{3 4 9 1}$ & 3937 & 11.33 & \\
\hline
\end{tabular}

\subsection{Classifiers Performance: Evaluation}

Two common methods to evaluate classifiers performance over the test set are:

1. Based on the sensitivity, specificity, and accuracy values (confusion matrix).

2. Based on receiver operating characteristic (ROC) and area under the ROC curve (AUC).

We evaluate classifiers using both methods next.

3.3.1. Classification Performance Based on the Sensitivity, Specificity, and Accuracy Values (Confusion Matrix)

With sensitivity, specificity and accuracy criteria, one can evaluate a classifier system based on correct and wrong classified samples, detailing between which classes errors are taken place if needed. Sensitivity, specificity, and accuracy are defined next in Equations (2)-(4):

$$
\begin{gathered}
\text { Sensitivity }=\frac{\mathrm{TP}}{\mathrm{TP}+\mathrm{FN}} \\
\text { Specificity }=\frac{\mathrm{TN}}{\mathrm{FP}+\mathrm{TN}} \\
\text { Accuracy }=\frac{\mathrm{TP}+\mathrm{TN}}{\mathrm{TP}+\mathrm{TN}+\mathrm{FP}+\mathrm{FN}}
\end{gathered}
$$

where true-positive fraction (TP) is equal to the number of samples in each class that are correctly classified, true-negative fraction (TN) is equal to the number of samples on main diagonal in confusion matrix (square matrix that has dimensions equal to number of classes, cf. Tables 7-9) minus the number of samples that are correctly classified in the intended class, false-negative fraction (FN) is defined as the sum of horizontal samples in the investigated class minus number of samples that are correctly classified in the intended class, and false-positive fraction (FP) is defined as the sum of vertical samples of the investigated class minus the number of samples that are correctly classified in the intended class (Wisaeng [17]). In short, a sensitivity value of $100 \%$ in a certain class means that all samples of this class were classified correctly; a specificity value of $100 \%$ in a certain class means that no samples from other classes were misclassified in that given class; and an accuracy value of $100 \%$ means that neither no samples from other classes were misclassified in a certain class nor any samples from that certain class were misclassified in other classes.

Table 10 shows classification performance criteria including specificity, sensitivity and accuracy results for 100 uniform random iterations using hybrid ANN-ABC, hybrid ANN-BBO and LDA 
classifiers. By looking carefully at Table 10, one realizes that the LDA classifier is in general terms superior to both ANN-ABC (slightly) and ANN-BBO (clearly), that red river gum tree class is consistently easier to classify among all three classifiers, and that opposite situation happens with $\mathrm{mt}$. Atlas mastic tree class. In precise terms, the following results are reached: best classifier, mean sensitivity, and mean specificity, were computed for the five trees under study, resulting in 1-Cydonia oblonga (quince) 95.89\% (ANN-ABC), 95.91\% (ANN-ABC); 2-Eucalyptus camaldulensis dehn (river red gum) 100\% (LDA), 100\% (LDA); 3-Malus pumila (apple) 96.63\% (LDA), 94.99\% (LDA); 4-Pistacia atlantica (mt. Atlas mastic tree), 91.71\% (LDA), 82.57\% (LDA); and 5-Prunus armeniaca (apricot) $88.67 \%$ (LDA), 94.65\% (LDA), respectively, all over the test set.

Table 10. Performance classification criteria results for ANN-ABC, ANN-BBO and LDA classifiers: sensitivity, accuracy and specificity, evaluated on 100 random train and test set samples, computed over the test set.

\begin{tabular}{cccccccccc}
\hline & \multicolumn{3}{c}{ Hybrid ANN-ABC } & \multicolumn{3}{c}{ Hybrid ANN-BBO } & \multicolumn{3}{c}{ LDA } \\
\cline { 2 - 9 } & sen $^{\mathbf{1}} \mathbf{( \% )}$ & acc $^{\mathbf{2}} \mathbf{( \% )}$ & $\mathbf{s p e}^{\mathbf{3}} \mathbf{( \% )}$ & sen (\%) & acc (\%) & spe (\%) & sen (\%) & acc (\%) & spe (\%) \\
\hline $\begin{array}{c}\text { 1-Cydonia } \\
\text { oblonga }\end{array}$ & 95.89 & 98.16 & 95.91 & 92.33 & 95.49 & 88.65 & 91.86 & 97.40 & 96.43 \\
$\begin{array}{c}\text { 2-Eucalyptus } \\
\text { camaldulensis d. }\end{array}$ & 99.64 & 99.70 & 99.13 & 94.60 & 97.71 & 95.90 & 100 & 100 & 100 \\
$\begin{array}{c}\text { 3-Malus pumila } \\
\text { 4-Pistacia }\end{array}$ & 93.61 & 97.61 & 94.56 & 87.62 & 95.24 & 85.55 & 96.63 & 98.26 & 94.99 \\
$\begin{array}{c}\text { atlantica } \\
\text { 5-Prunus } \\
\text { armeniaca }\end{array}$ & 87.21 & 95.43 & 86.57 & 82.19 & 93.82 & 83.25 & 91.71 & 95.20 & 82.57 \\
\hline
\end{tabular}

1. Sensitivity (sen), 2. Accuracy (acc), 3. Specificity (spe); 1-Cydonia oblonga (quince), 2-Eucalyptus camaldulensis dehn (river red gum), 3-Malus pumila (apple), 4-Pistacia atlantica (mt. Atlas mastic tree) and 5-Prunus armeniaca (apricot).

\subsubsection{Classification Performance Based on ROC and AUC}

Figure 7 shows ROC graphs for the different classes and different classifiers considered. In short, when plotting ROC curves, we are plotting FPR versus TPR, on the specificity-sensitivity plane. The higher the AUC values is the higher the classifier accuracy, while having an $A U C=1 / 2$ value is totally useless and simply equivalent to a random toss of a coin. As one can see, the ROC graphs related to hybrid ANN-ABC and LDA classifiers are near the upright end, so this implies that these two classifiers have good accuracy. At the same time, in Table 11 we show the mean and standard deviation of AUC values for the five different classes and three classifiers under consideration, over 100 repeated uniform random iterations. Figure 8 shows AUC boxplot graphs for 100 iterations based on ANN-ABC, $\mathrm{ANN}-\mathrm{BBO}$ and LDA classifiers. As one can see, these three classifiers classified red river gum class samples with extremely high accuracy, since boxplot graphs are very compact. Also, LDA if often ahead of its partners, closely followed by ANN-ABC, in terms of AUC values (please note that vertical axis AUC ranges are different in each figure). In precise terms, mean AUC values were computed for the five trees under study, resulting in 1-Cydonia oblonga (quince) 0.991 (ANN-ABC); 2-Eucalyptus camaldulensis dehn (river red gum) 1.00 (LDA); 3-Malus pumila (apple) 0.996 (LDA); 4-Pistacia atlantica (mt. Atlas mastic tree) 0.979 (LDA); and 5-Prunus armeniaca (apricot) 0.994 (LDA), respectively, all over the test set. 


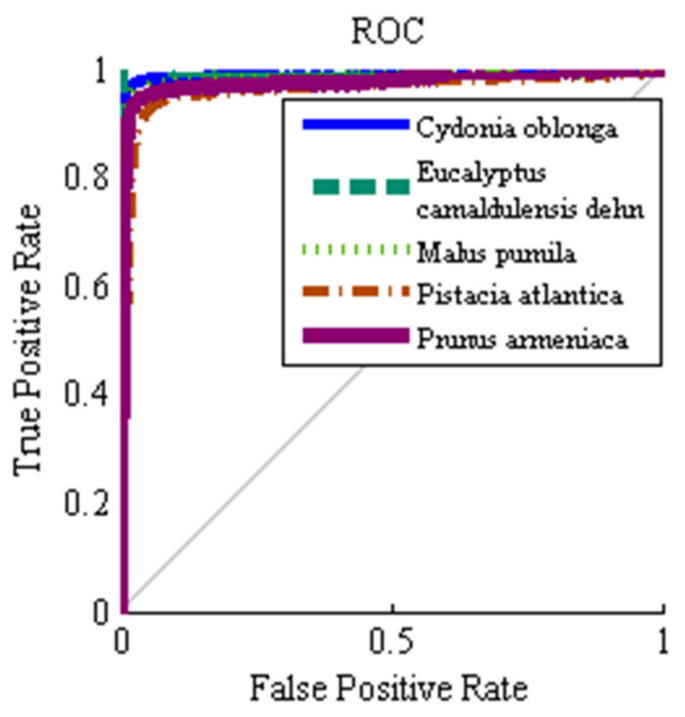

(a)

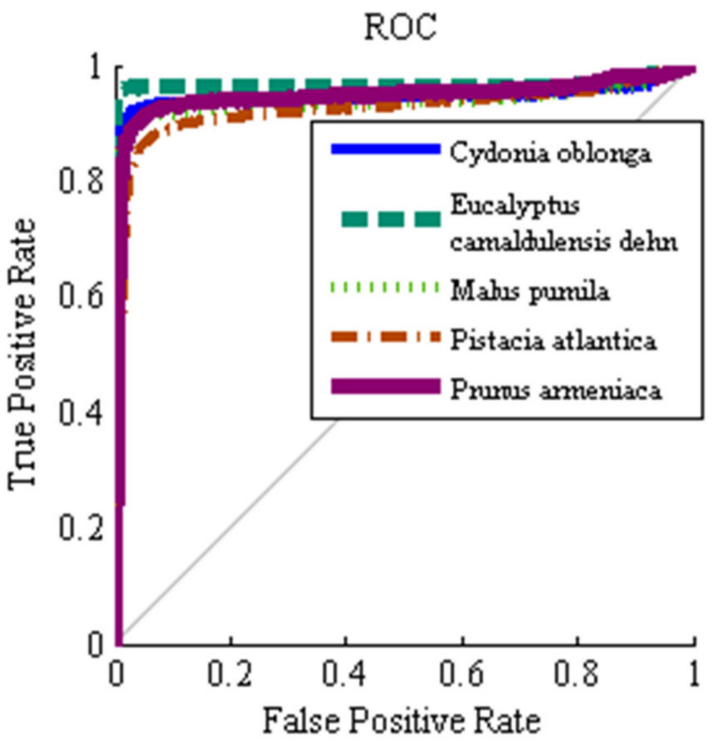

(b)

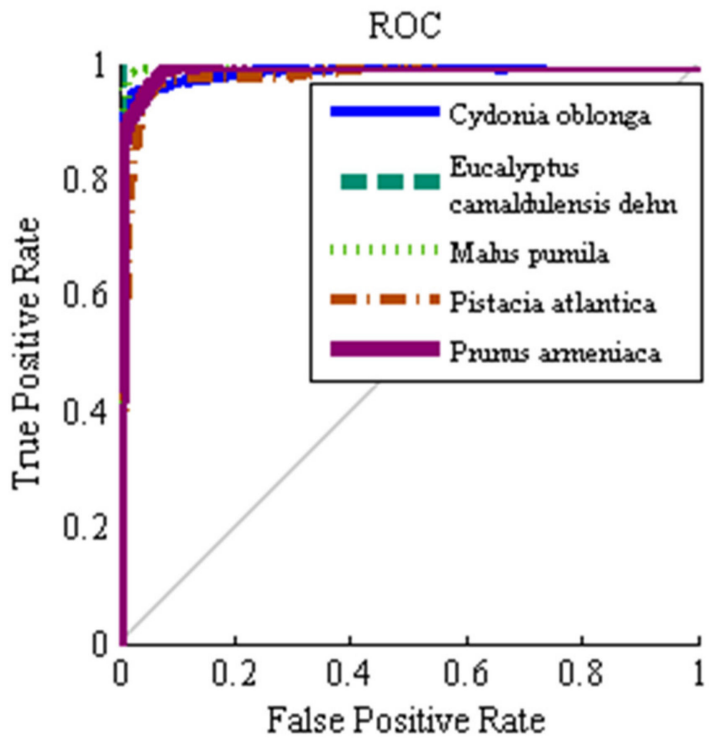

(c)

Figure 7. Average receiver operating characteristic (ROC) plots: (a) ANN-ABC, (b) ANN-BBO, and (c) LDA, classifiers. 1-Cydonia oblonga (quince), 2-Eucalyptus camaldulensis dehn (river red gum), 3-Malus pumila (apple), 4-Pistacia atlantica (mt. Atlas mastic tree) and 5-Prunus armeniaca (apricot). 100 random train and test set samples simulations averaged, computed over the test set.

Table 11. Mean \pm standard deviation area under the ROC curve (AUC) values for ANN-ABC, ANN-BBO and LDA classifiers: 100 random train and test set samples, computed over the test set.

\begin{tabular}{cccccc}
\hline & \multicolumn{5}{c}{ AUC (Mean \pm Standard Deviation) } \\
\cline { 2 - 6 } & Quince & Red Gum & Apple & Mastic & Apricot \\
\hline ANN-ABC & $0.991 \pm 0.010$ & $0.999 \pm 0.000$ & $0.985 \pm 0.017$ & $0.963 \pm 0.025$ & $0.986 \pm 0.015$ \\
ANN-BBO & $0.961 \pm 0.136$ & $0.968 \pm 0.139$ & $0.945 \pm 0.162$ & $0.945 \pm 0.114$ & $0.947 \pm 0.179$ \\
LDA & $0.988 \pm 0.005$ & $1.000 \pm 0.000$ & $0.996 \pm 0.002$ & $0.979 \pm 0.009$ & $0.994 \pm 0.003$ \\
\hline
\end{tabular}




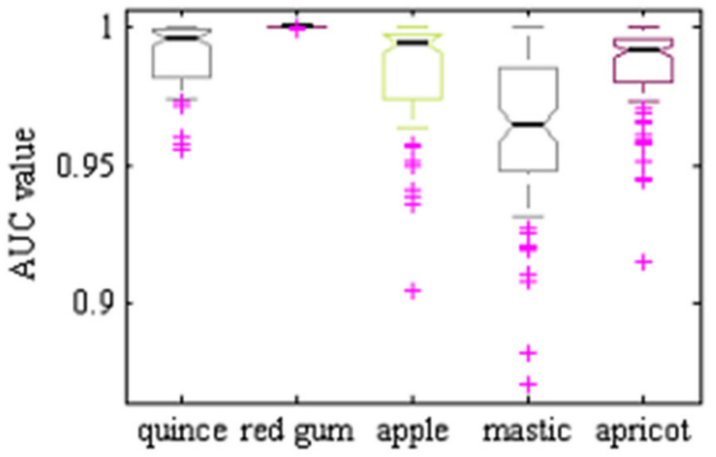

(a)

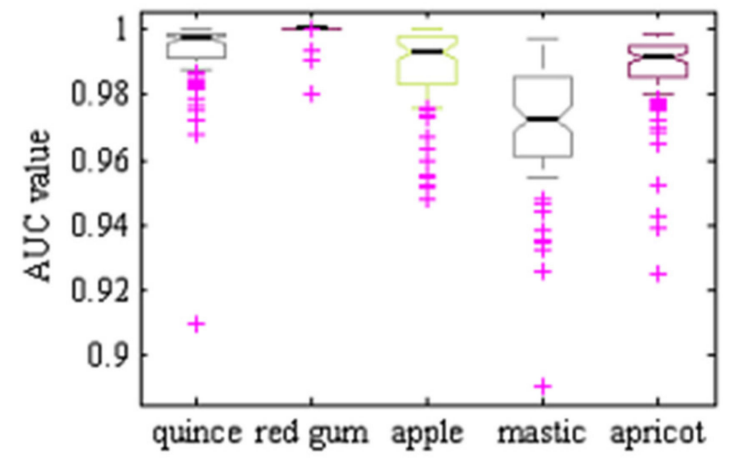

(b)

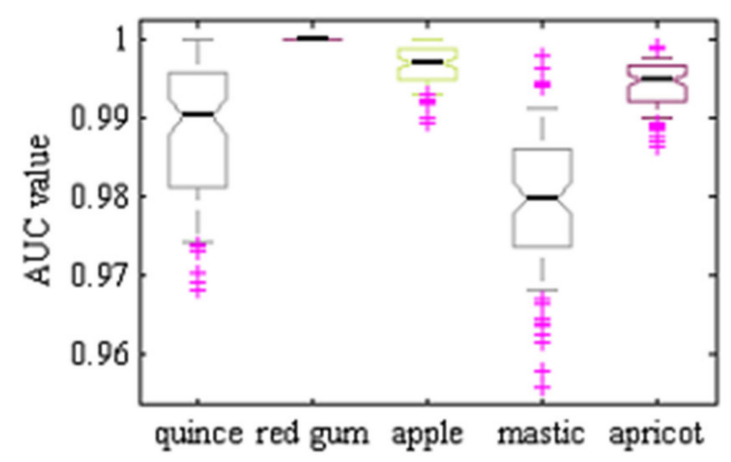

(c)

Figure 8. AUC boxplot graphs: (a) ANN-ABC, (b) ANN-BBO, and (c) LDA, classifiers. 1-Cydonia oblonga (quince), 2-Eucalyptus camaldulensis dehn (river red gum), 3-Malus pumila (apple), 4-Pistacia atlantica (mt. Atlas mastic tree) and 5-Prunus armeniaca (apricot). 100 random train and test set samples simulations averaged, computed over the test set.

Finally, to compare the CCR in this study with others, Singh and Bhamrah [2], Kadir [3], Mursalin et al., [5] and Ehsanirad [4], were used. It should be noted that since studies do not share the same leave image database, no direct comparison is possible. Singh and Bhamrah [2] classified 8 species of different plants with artificial neural network classifier and Kadir [3] used a Bayes classifier to classify some leaves related to several plants. Mursalin et al., [5] used some shape and color features for plant classification purposes. Ehsanirad [4] used only some texture features for the classification of different plants. Table 12 shows the accuracy or CCR of the different classification methods for comparison purposes. As one can see, the proposed method has a higher number of samples and higher accuracy than the other methods being compared with.

Table 12. Comparison of accuracy or the correct classification rate (CCR) of the different classification systems.

\begin{tabular}{ccc}
\hline Method & The Number of Samples & Correct Classification Rate (\%) \\
\hline Best result of here proposed model & 516 & 99.52 (test set) \\
Singh and Bhamrah [2] & 80 & 98.8 \\
Kadir [3] & 100 & 88 \\
Mursalin et al. [5] & 400 & 98.24 \\
Ehsanirad [4] & 65 & 78 \\
\hline
\end{tabular}


One possible reason for the superiority of the proposed method might be the use of different properties. As can be seen in Section 2.3, feature extraction, in this study several features comprising five different feature types were extracted. That can be of help in finding effective discriminative features that can properly separate different plant type leaves. While on the contrary, other researchers often used only a couple of feature types, such as color and texture features. A second possible reason for higher accuracy might refer to the structure of the neural network since the study here proposed the optimal structure of the ANN is adjusted by both $\mathrm{ABC}$ and $\mathrm{BBO}$ algorithms.

\section{Conclusions}

This study aims to design an imaging computer vision system to classify five types of tree leaf images. Relevant results can be summarized as follows to conclude:

1. Tree leaves images had the lowest level of noise (noise pixels) after segmentation in $\mathrm{YCbCr}$ color space, in comparison with RGB, YIQ, CMY, HSV and HSI color spaces.

2. Two channels in $\mathrm{YCbCr}$ color space were needed to image thresholding (a luminance and a chrominance channels).

3. Among the seven discriminant features selected, four features were texture features based on the gray level co-occurrence matrix and three features were shape features.

4. We believe that since samples in 2-Eucalyptus camaldulensis dehn (river red gum, class 2) have clear different shapes, shape features were selected as effective features, reaching almost no classification errors inside 2-Eucalyptus camaldulensis dehn (river red gum) class.

5. Whenever features in different classes do not present overlapping, the statistical LDA method usually has a superior performance.

Author Contributions: Conceptualization, S.S. R.P. and J.I.A.; methodology, S.S. and J.I.A.; software, S.S.; validation, S.S. and J.I.A.; formal analysis, S.S. and J.I.A.; investigation, S.S. and J.I.A.; resources, S.S.; data curation, J.I.A.; writing — original draft preparation, J.I.A.; writing — review and editing, S.S., R.P. and J.I.A.; visualization, S.S. and R.P.; supervision, J.I.A.; project administration, J.I.A. All authors have read and agreed to the published version of the manuscript.

Funding: This research was funded in part by the European Union (EU) under the Erasmus+ project entitled "Fostering Internationalization in Agricultural Engineering in Iran and Russia" [FARmER] with grant number 585596-EPP-1-2017-1-DE-EPPKA2-CBHE-JP.

Conflicts of Interest: The authors declare no conflict of interests.

\section{Appendix A}

We depict next all five type 516 segmented tree leaves image database, as shown in Figures A1-A5, corresponding to, 1-Cydonia oblonga (quince), 2-Eucalyptus camaldulensis dehn (river red gum), 3-Malus pumila (apple), 4-Pistacia atlantica ( $\mathrm{mt}$. Atlas mastic tree) and 5-Prunus armeniaca (apricot), respectively. 


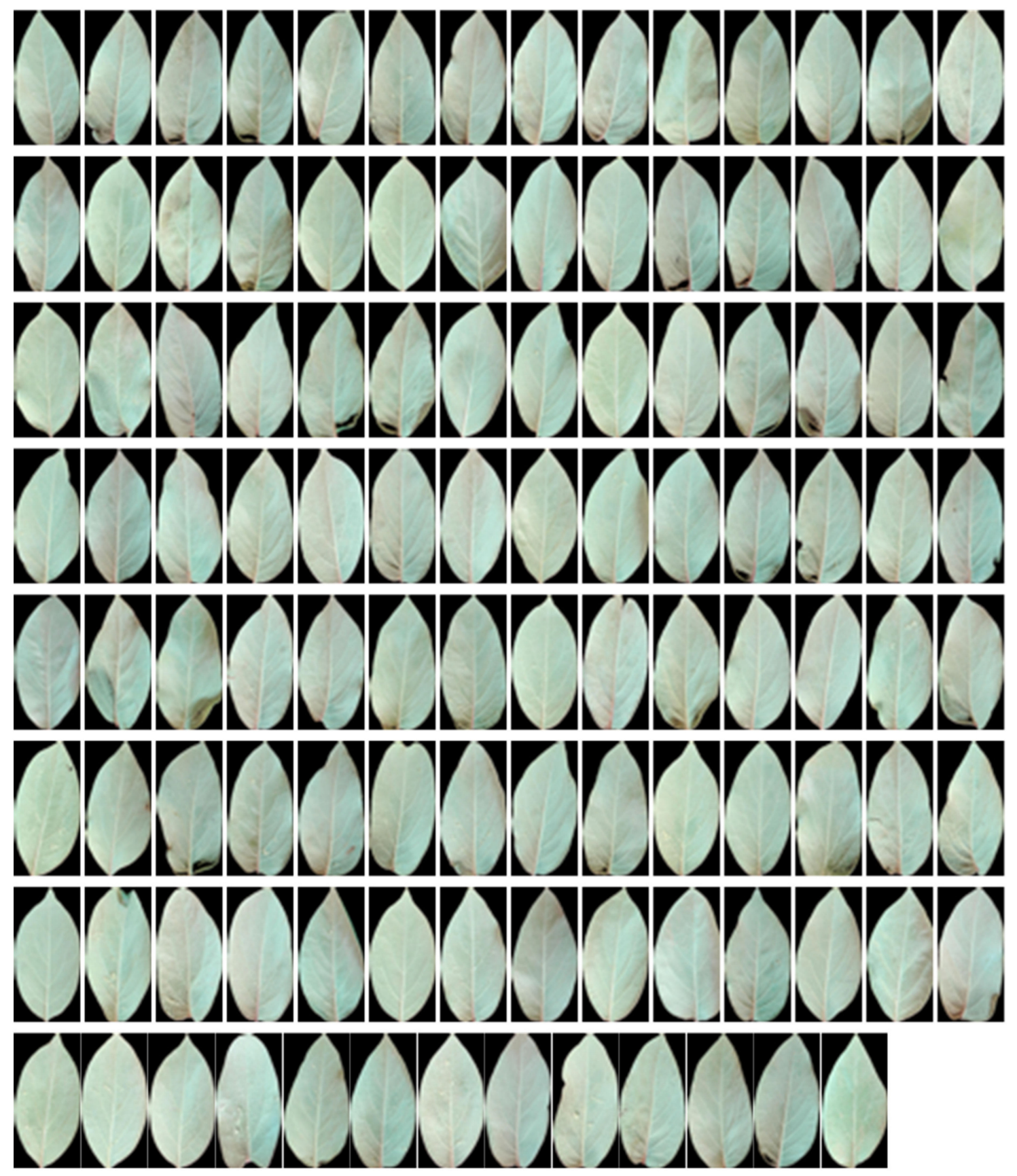

Figure A1. Cydonia oblonga (quince) tree leaves image database: 111 samples. 

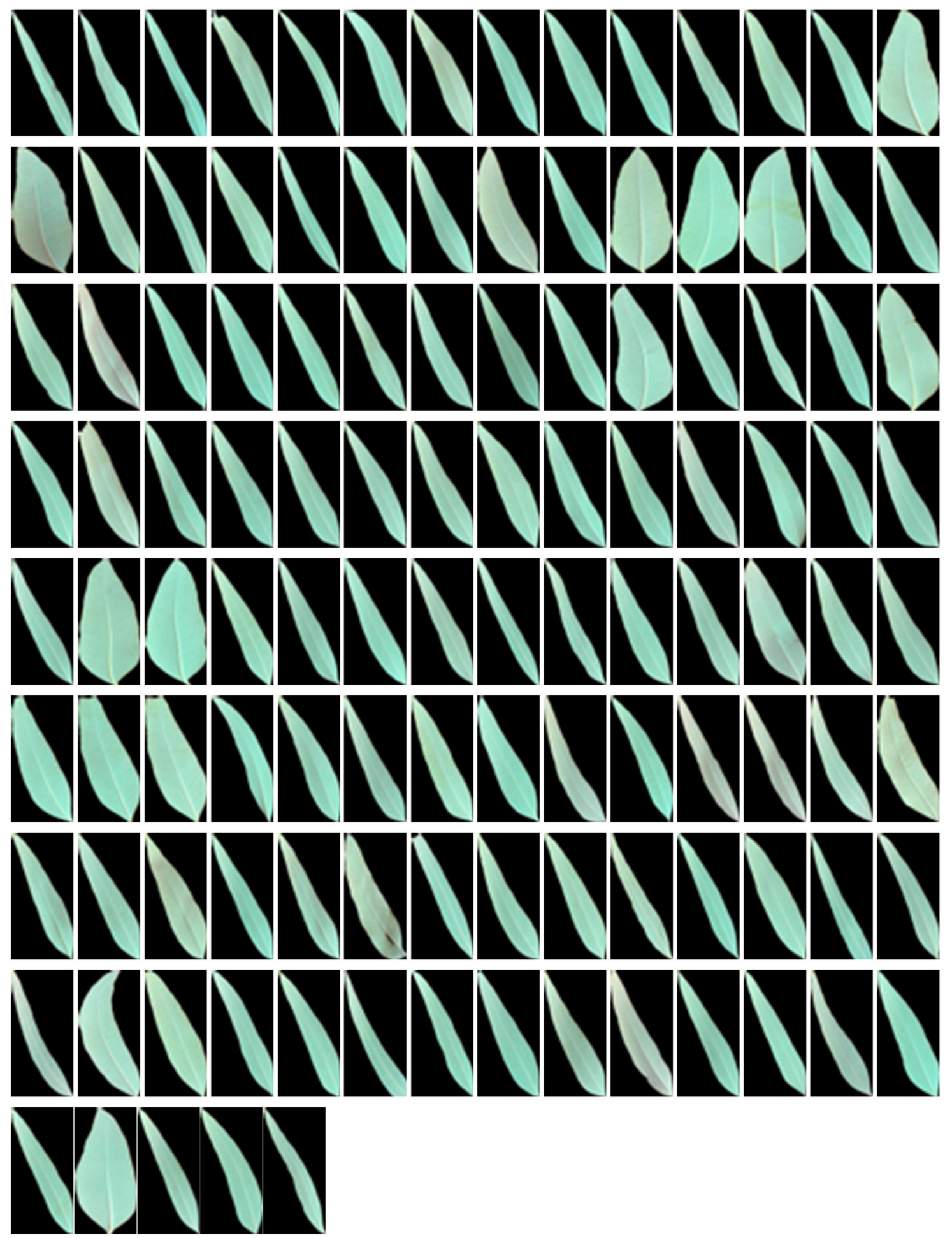

Figure A2. Eucalyptus camaldulensis dehn (river red gum) tree leaves image database: 117 samples. 


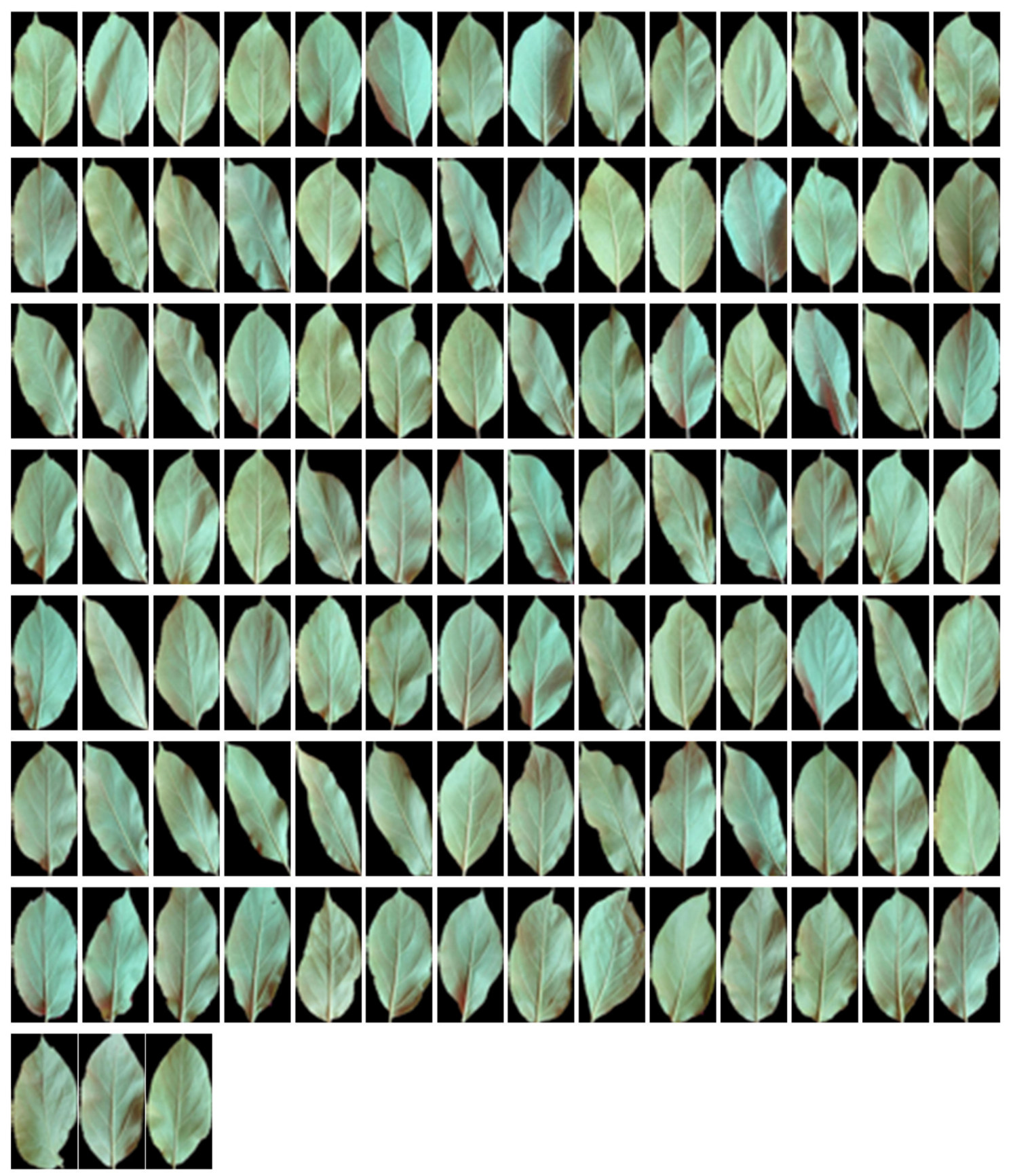

Figure A3. Malus pumila (apple) tree leaves image database: 101 samples. 


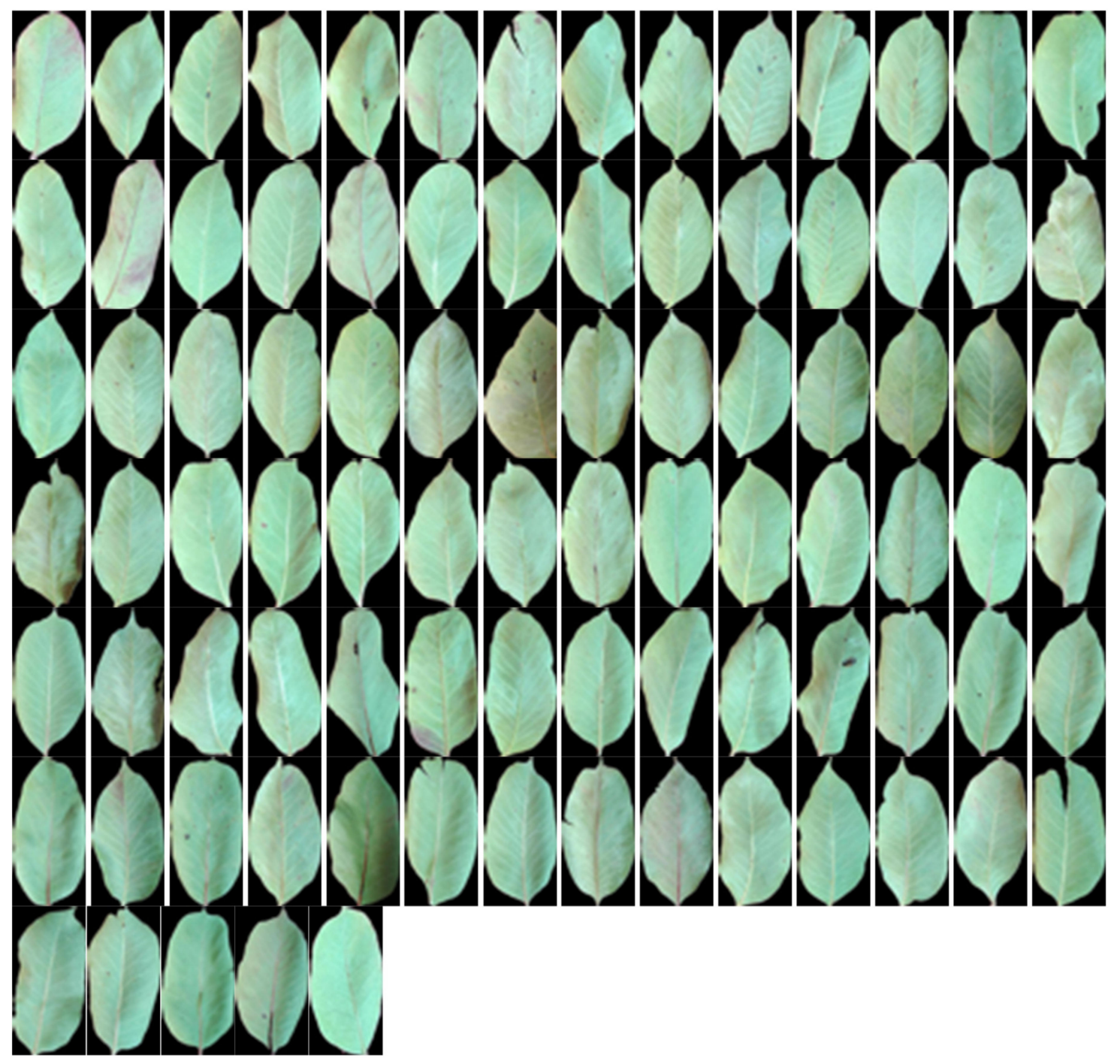

Figure A4. Pistacia atlantica (mt. Atlas mastic tree) tree leaves image database: 89 samples. 


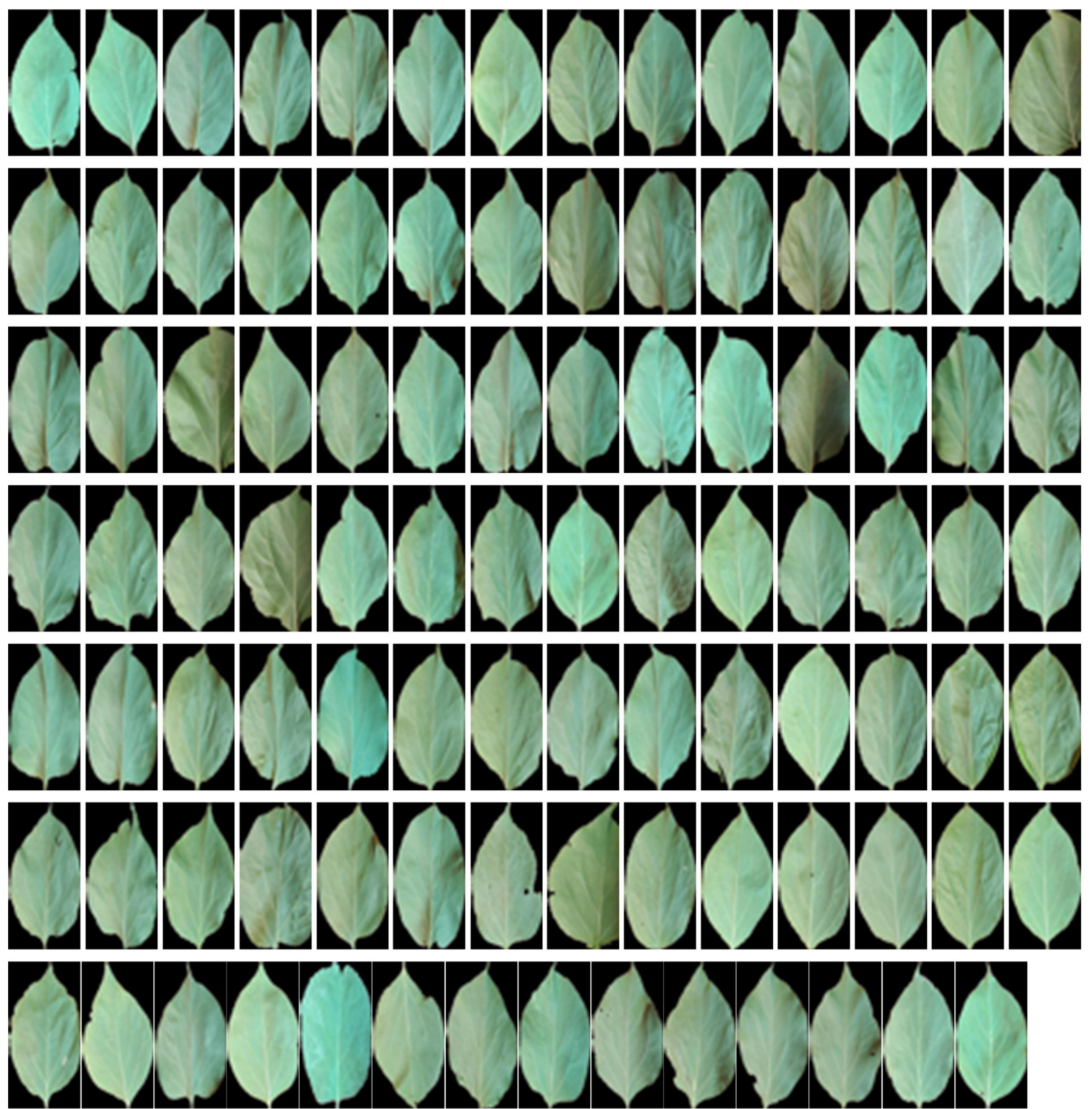

Figure A5. Prunus armeniaca (apricot) tree leaves image database: 98 samples.

\section{References}

1. Liu, F.H.; O'Connell, N.V. Off-site movement of surface-applied simazine from a citrus orchard as affected by irrigation incorporation. Weed Sci. 2002, 50, 672-676. [CrossRef]

2. Singh, S.; Bhamrah, M.S. Leaf identification using feature extraction and neural network. IOSR J. Electron. Commun. Eng. 2015, 10, 134-140.

3. Kadir, A. Leaf Identification Using Fourier Descriptors and Other Shape Features. Gate Comput. Vis. Pattern Recognit. 2015, 1, 3-7. [CrossRef]

4. Ehsanirad, A. Plant classification based on leaf recognition. Int. J. Comput. Sci. Inform. Sec. 2010, 8, 78-81.

5. Mursalin, M.; Hossain, M.; Noman, K.; Azam, S. Performance Analysis among Different Classifier Including Naive Bayes, Support Vector Machine and C4.5 for Automatic Weeds Classification. Glob. J. Comput. Sci. Technol. Graph. Vis. 2013, 13, 11-16.

6. Ahmed, F.; Al-Mamun, H.A.; Bari, A.S.M.H.; Hossain, E.; Kwan, P. Classification of crops and weeds from digital images: A support vector machine approach. Crop Prot. 2012, 40, 98-104. [CrossRef] 
7. Rumpf, T.; Römer, C.; Weis, M.; Sökefeld, M.; Gerhards, R.; Plümer, L. Sequential support vector machine classification for small-grain weed species discrimination with special regard to Cirsium arvense and Galium aparine. Comput. Electron. Agric. 2012, 80, 89-96. [CrossRef]

8. Pereira, L.A.M.; Nakamura, R.Y.M.; Souza, G.F.S.d.; Martins, D.; Papa, J.P. Aquatic weed automatic classification using machine learning techniques. Comput. Electron. Agric. 2012, 87, 56-63. [CrossRef]

9. Azlah, M.A.F.; Chua, L.S.; Rahmad, F.R.; Abdullah, F.I.; Wan Alwi, S.R. Review on Techniques for Plant Leaf Classification and Recognition. Computers 2019, 8, 77. [CrossRef]

10. Gonzalez, R.C.; Woods, R.E.; Eddins, S.L. Digital Image Processing Using MATLAB; Prentice Hall: Upper Saddle River, NY, USA, 2004.

11. Woebbecke, D.; Meyer, G.E.; Bargen, K.V.; Mortensen, D.A. Color indices for weed identification under various soil, residue, and lighting conditions. Trans. ASAE 1995, 3, 259-269. [CrossRef]

12. Meyer, G.E.; Mehta, T.; Kocher, M.F.; Mortensen, D.A.; Samal, A. Textural imaging and discriminant analysis for distinguishing weeds for spot spraying. Trans. ASAE 1998, 41, 1189-1197. [CrossRef]

13. Kataoka, T.; Kaneko, T.; Okamoto, H.; Hata, S. Crop growth estimation system using machine vision. In Proceedings of the IEEE/ASME International Conference Advances Intelligence Mechatronics (AIM 2003), Kobe, Japan, 20 July-24 July 24 2003; pp. 1079-1083.

14. Meyer, G.E.; Neto, J.A.C. Verification of color vegetation indices for automated crop imaging applications. Comput. Electron. Agric. 2008, 63, 282-293. [CrossRef]

15. Woebbecke, D.M.; Meyer, G.E.; Bargen, K.V.; Mortensen, D.A. Plant species identification, size, and enumeration using machine vision techniques on near-binary images. Opt. Agric. For. 1992, 1836, 208-219.

16. Golzarian, M.R.; Frick, R.A. Classification of images of wheat, ryegrass and brome grass species at early growth stages using principal component analysis. Plant Meth. 2011, 7, 7-28. [CrossRef] [PubMed]

17. Wisaeng, K. A Comparison of Decision Tree Algorithms For UCI Repository Classification. Int. J. Eng. Trends Technol. 2013, 4, 3393-3397.

(C) 2020 by the authors. Licensee MDPI, Basel, Switzerland. This article is an open access article distributed under the terms and conditions of the Creative Commons Attribution (CC BY) license (http://creativecommons.org/licenses/by/4.0/). 